\title{
THE PRINCIPLE OF MINIMIZED ITERATIONS IN THE SOLUTION OF THE MATRIX EIGENVALUE PROBLEM ${ }^{1}$
}

\author{
BY \\ W. E. ARNOLDI \\ Hamilton Standard Division, United Aircraft Corporation, East Hartford, Conn.
}

An interpretation of Dr. Cornelius Lanczos' iteration method, which he has named "minimized iterations", is discussed in this article, expounding the method as applied to the solution of the characteristic matrix equations both in homogeneous and nonhomogeneous form. This interpretation leads to a variation of the Lanczos procedure which may frequently be advantageous by virtue of reducing the volume of numerical work in practical applications. Both methods employ essentially the same algorithm, requiring the generation of a series of orthogonal functions through which a simple matrix equation of reduced order is established. The reduced matrix equation may be solved directly in terms of certain polynomial functions obtained in conjunction with the generated orthogonal functions, and the convergence of the solution may be observed as the order of the reduced matrix is successively increased with the order of the original matrix as a limit. The method of minimized iterations is recommended as a rapid means for determining a small number of the larger eigenvalues and modal columns of a large matrix and as a desirable alternative for various series expansions of the Fredholm problem.

1. The conventional iterative procedures. It is frequently required that real latent roots, or eigenvalues, and modal columns be determined for a real numerical matrix, $u$, of order, $n$, in the characteristic homogeneous equation, ${ }^{*}$

$$
(\lambda I-u) k=0
$$

which is satisfied by any of $n$ values of the scalar, $\lambda=\lambda_{r}$ with their associated modal columns, $k=k_{r}$. Beginning with an arbitrary column, $k_{0}$, and repeatedly premultiplying by $u$, the iterative procedure, $k_{i+1}=u k_{i}$, will converge to the modal column corresponding to the largest, or dominant, latent root. After obtaining this solution, the dominant mode may be removed by any of several methods, so that the next largest root of the original matrix becomes the dominant root of an altered matrix, whereupon the same procedure may be repeated to obtain the next root, and so on, until all desired roots and modal columns have been obtained. Since the accuracy of each root and modal column is dependent upon the accuracy with which each previous column has been determined, this method obviously requires a wasteful amount of labor if the initial roots and modes are not needed, as is often the case in practical applications, and convergence can be extremely slow if the roots are not widely dispersed.

The non-homogeneous equation, or Fredholm problem in matrix form, may be represented by

$$
(\lambda I-u) k=q
$$

${ }^{1}$ Received May 15, 1950.

*The general notation used in this discussion to represent matrices, columns, rows, and matrix equations follows as closely as possible the conventions established in "Elementary Matrices", Frazer, Duncan and Collar, Cambridge, 1938. Brackets and braces are employed only when necessary to avoid confusion, e.g., to distinguish between the column, $k_{i}$, and the matrix of $m$ such columns, $\left[k_{i}\right]$. 
where $\lambda$ and $q$ are specified and a solution is desired for $k$. This solution may be obtained by direct treatment of the array as simultaneous equations, a common algorithm being the method of pivotal condensation [1], a lengthy and laborious procedure but sometimes indispensable. Among essentially iterative methods are the Schmidt expansion [2], which requires first the solution for latent roots, modal columns, and modal rows, whereupon $k$ is found as

$$
k=\sum_{r=1}^{n} \frac{1}{\lambda-\lambda_{r}} \frac{\kappa_{r} q}{\kappa_{r} k_{r}} k_{r} \quad\left(\kappa_{r}=\text { modal row }\right) .
$$

Another iterative method is based upon the Liouville-Neumann expansion [2], where the reciprocal matrix is written as an infinite geometric series,

$$
k=(\lambda I-u)^{-1} q=\lambda^{-1}\left(I+\lambda^{-1} u+\lambda^{-2} u^{2}+\cdots\right) q
$$

whose solution is obtained by iteration:

$$
k_{i+1}=\lambda^{-1} q+\lambda^{-1} u k_{i}
$$

Unfortunately, this method converges only if $\lambda$ is greater in magnitude than the magnitude of the dominant root of $u$. It can also be applied in special cases when the field of roots can be shifted so as to meet the requirement for convergence, but the method is nevertheless of limited application and is often subject to slow convergence.

2. A classical method for reducing matrix order. The solution of the original homogeneous equation may be replaced by the solution of a matrix equation of reduced order by introducing the approximation that the modal column, $k$, may be represented by the sum of a series of arbitrary columns, $k_{i}$, multiplied by coefficients, $c_{2}$, to be established in the solution. Substituting

$$
k=\sum_{i=1}^{m} c_{i} k_{i} \equiv\left[k_{i}\right] c
$$

into Eq. (1) yields

$$
(\lambda I-u)\left[k_{i}\right] c=0,
$$

where $\left[k_{i}\right]$ is a rectangular matrix whose columns are $k_{i}$, and $c$ is a column of elements $c_{i}$. Now premultiply Eq. (4) by another rectangular matrix whose rows are $\kappa_{i}$, corresponding with $k_{i}$ in such fashion that the row $\kappa=\sum_{j=1}^{m} c_{j} \kappa_{j}$ will satisfy the original matrix in the form,

$$
\kappa(\lambda I-u)=0 .
$$

It should be noted that the functions, $k_{i}$ and $\kappa_{i}$, are also required to satisfy the point boundary conditions implicit in the matrix, $u$. The matrix equation now becomes

$$
\left[\kappa_{i}\right](\lambda I-u)\left[k_{i}\right] c=0,
$$

or, in more concise form,

$$
\left(\lambda I-\left[\kappa_{i} k_{i}\right]^{-1}\left[\kappa_{i} u k_{i}\right]\right) c=0 .
$$

Equation (5) will henceforth be referred to as the "reduced equation," and the matrix, $\left[\kappa_{i} k_{i}\right]^{-1}\left[\kappa_{j} u k_{i}\right]$, will be known as the "reduced matrix." Since $m$ functions were assumed to be sufficient for an approximate solution, this equation involves a matrix of $m$ order, 
presumably less than the original $n$ order, hence more rapidly solved than the original by conventional iterative procedures. If one could hope that $m$ could be chosen sufficiently small, a solution might be available by direct expansion of the determinant, but it is usually necessary, in the general form given above, to employ a relatively large number of functions, $k_{i}$ and $\kappa_{j}$, so that the advantages of this transformation of the original matrix are not universally apparent. Furthermore, the labor of calculating the elements of the reduced matrix may be great, particularly since the inversion of $\left[\kappa_{i} k_{i}\right]$ is required.

The Galerkin method [3] is a variation of the process outlined above, differing only in the choice of $\kappa_{j}$. By the Galerkin method, each $\kappa_{i}$ is the transposed of the corresponding column, $k_{i}$, which somewhat simplifies the determination of the inverse matrix required. However, it is also objectionable on the basis of tedium.

3. The Lanczos method of minimized iterations. The following exposition differs in form from the discussion originally given by Lanczos [2], but it arrives at the same results and makes a further extension of the Galerkin type more evident. Lanczos reduces the matrix order as described above but eliminates the objections of labor in the formation of the reduced matrix, indicates a solution in terms of polynomial equations derived from a direct iterative procedure involving the original matrix, avoids the necessity for separately forming and solving the reduced matrix, and provides a means for efficiently generating the required columns and rows in order that a convergent solution will be obtained with a minimum number.

To deduce the Lanczos method of minimized iterations, it is first observed that the formation of the reduced matrix would be greatly simplified if the matrix, $\left[\kappa_{j} k_{i}\right]$, were of diagonal form, the otherwise arbitrary rows and columns initially chosen being of such form as to satisfy $\kappa_{j} k_{i}=0$ when $i \neq j$. The inverse would then consist of a diagonal matrix whose individual elements would be the reciprocals of the corresponding elements in $\left[\kappa_{i} k_{i}\right]$, and the reduced equation would take the form

$$
\left(\lambda I-\left[\frac{\kappa_{j} u k_{i}}{\kappa_{j} k_{j}}\right]\right) c=0 .
$$

This form has further potential advantages, obtained by observing that the desired orthogonality relationship among the chosen rows and columns may be established by generating these rows and columns from an initial row and column according to the following expressions:

$$
\begin{aligned}
& k_{i+1}=u k_{i}-\alpha_{i} k_{i}-\beta_{i-1} k_{i-1}-\gamma_{i-2} k_{i-2}-\delta_{i-3} k_{i-3}-\cdots, \\
& \kappa_{i+1}=\kappa_{i} u-A_{i} \kappa_{i}-B_{i-1} \kappa_{i-1}-C_{i-2} \kappa_{i-2}-D_{i-3} \kappa_{i-3}-\cdots .
\end{aligned}
$$

The necessary scalar constants, $\alpha_{i}, \beta_{i-1}, \gamma_{i-2}, \cdots, A_{i}, B_{i-1}, C_{i-2}, \cdots$, may be defined by forming certain scalars as row-column products and noting that many elements vanish, due to the orthogonality relation postulated, $\kappa_{1} k_{i}=0$ for $i \neq j$, as follows:

$$
\begin{aligned}
& \kappa_{i} k_{i+1}=\kappa_{i} u k_{i}-\alpha_{i} \kappa_{i} k_{i}=0, \\
& \kappa_{i} k_{i+2}=\kappa_{i} u k_{i+1}-\beta_{i} \kappa_{i} k_{i}=0, \\
& \kappa_{i} k_{i+3}=\kappa_{i} u k_{i+2}-\gamma_{i} \kappa_{i} k_{i}=0,
\end{aligned}
$$

etc. 
also

$$
\begin{gathered}
\kappa_{i+1} k_{i}=\kappa_{i} u k_{i}-A_{i} \kappa_{i} k_{i}=0, \\
\kappa_{i+2} k_{i}=\kappa_{i+1} u k_{i}-B_{i} \kappa_{i} k_{i}=0 \\
\kappa_{i+3} k_{i}=\kappa_{i+2} u k_{i}-C_{i} \kappa_{i} k_{i}=0 \\
\text { etc. }
\end{gathered}
$$

whence

$$
\begin{array}{llll}
\alpha_{i}=\frac{\kappa_{i} u k_{i}}{\kappa_{i} k_{i}}, & \beta_{i}=\frac{\kappa_{i} u k_{i+1}}{\kappa_{i} k_{i}}, & \gamma_{i}=\frac{\kappa_{i} u k_{i+2}}{\kappa_{i} k_{i}}, & \text { etc. } \\
A_{i}=\frac{\kappa_{i} u k_{i}}{\kappa_{i} k_{i}}, & B_{i}=\frac{\kappa_{i+1} u k_{i}}{\kappa_{i} k_{i}}, & C_{i}=\frac{\kappa_{i+2} u k_{i}}{\kappa_{i} k_{i}}, & \text { etc. }
\end{array}
$$

Note that $\alpha_{i}=A_{i}$. Other identities may be found as follows:

$$
\begin{aligned}
u k_{i} & =k_{i+1}+\alpha_{i} k_{i}+\beta_{i-1} k_{i-1}+\cdots, \\
\kappa_{i+1} u k_{i} & =\kappa_{i+1} k_{i+1} .
\end{aligned}
$$

Also,

$$
\begin{aligned}
\kappa_{i} u & =\kappa_{i+1}+A_{i} \kappa_{i}+B_{i-1} \kappa_{i-1}+\cdots, \\
\kappa_{i} u k_{i+1} & =\kappa_{i+1} k_{i+1} .
\end{aligned}
$$

Therefore,

$$
\beta_{i}=B_{i} .
$$

The later constants will vanish, as is next shown.

$$
\kappa_{i} u=\kappa_{i+1}+A_{i} \kappa_{i}+B_{i-1} \kappa_{i-1}+C_{i-2} \kappa_{i-2}+\cdots .
$$

Therefore,

$$
\kappa_{i} u k_{i+2}=0
$$

whence,

$$
\gamma_{i}=0 \text {. }
$$

Similarly, $C_{i}=0$, and all further scalars, $\delta_{i}, \epsilon_{i}, \cdots, D_{i}, E_{2}, \cdots$, are also found to vanish.

The functional relationships by means of which the desired orthogonal rows and columns are generated thus become

$$
\begin{aligned}
& k_{i+1}=u k_{i}-\alpha_{i} k_{i}-\beta_{i-1} k_{i-1}, \\
& \kappa_{i+1}=\kappa_{i} u-\alpha_{i} \kappa_{i}-\beta_{i-1} \kappa_{i-1},
\end{aligned}
$$


where

$$
\alpha_{i}=\frac{\kappa_{i} u k_{i}}{\kappa_{i} k_{i}}, \quad \beta_{i-1}=\frac{\kappa_{i-1} u k_{i}}{\kappa_{i-1} k_{i-1}}
$$

The pertinent scalars and zeros having been established, it is now convenient to identify the elements of the reduced matrix, outlined as follows:

$$
\left[\frac{\kappa_{j} u k_{i}}{\kappa_{i} k_{i}}\right] \equiv\left\|\begin{array}{|cccccc}
\frac{\kappa_{1} u k_{1}}{\kappa_{1} k_{1}} & \frac{\kappa_{1} u k_{2}}{\kappa_{1} k_{1}} & \frac{\kappa_{1} u k_{3}}{\kappa_{1} k_{1}} & \frac{\kappa_{1} u k_{4}}{\kappa_{1} k_{1}} & \ldots & \frac{\kappa_{1} u k_{m}}{\kappa_{1} k_{1}} \\
\frac{\kappa_{2} u k_{1}}{\kappa_{2} k_{2}} & \frac{\kappa_{2} u k_{2}}{\kappa_{2} k_{2}} & \frac{\kappa_{2} u k_{3}}{\kappa_{2} k_{2}} & \frac{\kappa_{2} u k_{4}}{\kappa_{2} k_{2}} & \ldots & \frac{\kappa_{2} u k_{m}}{\kappa_{2} k_{2}} \\
\frac{\kappa_{3} u k_{1}}{\kappa_{3} k_{3}} & \frac{\kappa_{3} u k_{2}}{\kappa_{3} k_{3}} & \frac{\kappa_{3} u k_{3}}{\kappa_{3} k_{3}} & \frac{\kappa_{3} u k_{4}}{\kappa_{3} k_{3}} & \ldots & \frac{\kappa_{3} u k_{m}}{\kappa_{3} k_{3}} \\
\frac{\kappa_{4} u k_{1}}{\kappa_{4} k_{4}} & \frac{\kappa_{4} u k_{2}}{\kappa_{4} k_{4}} & \frac{\kappa_{4} u k_{3}}{\kappa_{4} k_{4}} & \frac{\kappa_{4} u k_{4}}{\kappa_{4} k_{4}} & \ldots & \frac{\kappa_{4} u k_{m}}{\kappa_{4} k_{4}} \\
\ldots \ldots & \ldots \ldots & \ldots \ldots & \ldots \ldots & \ldots & \ldots \\
\frac{\kappa_{m} u k_{1}}{\kappa_{m} k_{m}} & \frac{\kappa_{m} u k_{2}}{\kappa_{m} k_{m}} & \frac{\kappa_{m} u k_{3}}{\kappa_{m} k_{m}} & \frac{\kappa_{m} u k_{4}}{\kappa_{m} k_{m}} & \ldots & \frac{\kappa_{m} u k_{m}}{\kappa_{m} k_{m}}
\end{array}\right\|
$$

The elements are easily recognized and replaced, yielding

$$
\left[\frac{\kappa_{j} u k_{i}}{\kappa_{j} k_{i}}\right] \equiv\left\|\begin{array}{cccccc}
\alpha_{1} & \beta_{1} & 0 & 0 & \cdots & 0 \\
1 & \alpha_{2} & \beta_{2} & 0 & \cdots & 0 \\
0 & 1 & \alpha_{3} & \beta_{3} & \cdots & 0 \\
0 & 0 & 1 & \alpha_{4} & \cdots & 0 \\
\cdots & \cdots & \cdots & \cdots & \cdots & \cdots \\
0 & 0 & 0 & 0 & \cdots & \alpha_{m}
\end{array}\right\| .
$$

The vanishing of so many elements makes the expansion of the characteristic determinant extremely simple, so that a treatment of the reduced matrix equation by conventional iterative methods is unnecessary, and the step from the formation of the orthogonal row and column sequence to the solution for roots and modal columns may be taken without specifically examining the reduced matrix. The determinant will be expanded in stages, beginning with first order, which corresponds to the choice of one row and column, and successively increasing the order until the desired number of rows and columns has been reached. The resulting polynomials, individually equated to zero, will 
have roots which will represent successive approximations to the roots of the original matrix. These polynomials, which may be written by inspection, follow:

The determinant is

$$
\left|\lambda I-\left[\frac{\kappa_{i} u k_{i}}{\kappa_{i} k_{i}}\right]\right|=\left|\begin{array}{cccc}
\lambda-\alpha_{1} & -\beta_{1} & 0 & \cdots \\
-1 & \lambda-\alpha_{2} & -\beta_{2} & \ldots \\
0 & -1 & \lambda-\alpha_{3} & \ldots \\
\ldots \ldots \ldots \ldots \ldots \ldots \ldots \ldots \ldots
\end{array}\right|=p_{m}(\lambda) .
$$

The polynomials are

$$
\begin{aligned}
p_{1}(\lambda) & =\lambda-\alpha_{1}, \\
p_{2}(\lambda) & =\left(\lambda-\alpha_{2}\right) p_{1}(\lambda)-\beta_{1}, \\
p_{3}(\lambda) & =\left(\lambda-\alpha_{3}\right) p_{2}(\lambda)-\beta_{2} p_{1}(\lambda), \\
p_{4}(\lambda) & =\left(\lambda-\alpha_{4}\right) p_{3}(\lambda)-\beta_{3} p_{2}(\lambda), \\
p_{i+1}(\lambda) & =\left(\lambda-\alpha_{i+1}\right) p_{i}(\lambda)-\beta_{i} p_{i-1}(\lambda) .
\end{aligned}
$$

The solution for the modal column corresponding to a root of $p_{m}(\lambda)=0$ will be available after the coefficients, $c_{i}$, have been obtained by direct solution of the reduced matrix equation as a set of simultaneous scalar equations. Since one coefficient may be arbitrary, let $c_{1}=1$. Then,

$$
\begin{gathered}
c_{2}=\frac{\lambda-\alpha_{1}}{\beta_{1}}=\frac{p_{1}(\lambda)}{\beta_{1}}, \\
c_{3}=\frac{\left(\lambda-\alpha_{2}\right) c_{2}-1}{\beta_{2}}=\frac{p_{2}(\lambda)}{\beta_{1} \beta_{2}}, \\
c_{4}=\frac{\left(\lambda-\alpha_{3}\right) c_{3}-c_{2}}{\beta_{3}}=\frac{p_{3}(\lambda)}{\beta_{1} \beta_{2} \beta_{3}}, \\
c_{i+1}=\frac{\left(\lambda-\alpha_{i}\right) c_{i}-c_{i-1}}{\beta_{i}}=\frac{p_{i}(\lambda)}{\beta_{1} \beta_{2} \cdots \beta_{i}},
\end{gathered}
$$

and we have the modal column through its initial definition,

$$
k=\sum_{i=1}^{m} c_{i} k_{i}=k_{1}+\frac{p_{1}(\lambda)}{\beta_{1}} k_{2}+\frac{p_{2}(\lambda)}{\beta_{1} \beta_{2}} k_{3}+\cdots+\frac{p_{m-1}(\lambda)}{\beta_{1} \beta_{2} \cdots \beta_{m-1}} k_{m} .
$$

It is important to note that, not only is this a finite series, but it is limited in length to a maximum number of terms, $m=n$, the order of the original matrix. The generation of the orthogonal function sequence stops at this point, since the original matrix on 
which it is based is incapable of defining more than $n$ linearly independent functions, and when this number has been reached the reduced matrix will yield precisely the roots and modal columns of the original matrix. This characteristic, more fully discussed by Lanczos [2], is an important feature of the method of minimized iterations, since it indicates that this method, unlike the conventional matrix iteration algorithm, will never require a continuation ad infinitum.

4. Minimized iterations for the non-homogeneous equation. Given the equation

$$
(\lambda I-u) k=q,
$$

where $\lambda$ and $q$ are specified and a solution for $k$ is desired, the reduced equation is formed exactly as for the homogeneous equation, replacing $k$ and premultiplying by $\left[\kappa_{i}\right]$ as follows:

$$
\left(\lambda I-\left[\frac{\kappa_{i} u k_{i}}{\kappa_{j} k_{i}}\right]\right) c=\left[\frac{\kappa_{i}}{\kappa_{j} k_{i}}\right] q .
$$

The column on the right side of the equation is vastly simplified by letting $k_{1}=q$ whence

$$
\left(\lambda I-\left[\frac{\kappa_{i} u k_{i}}{\kappa_{j} k_{i}}\right]\right) c=\{1,0,0, \cdots 0\} .
$$

The method of minimized iterations is now employed to determine the same scalars, $\alpha_{i}$ and $\beta_{i}$, as before, whereupon the solution for $c$ is indicated as follows:

$$
c=\left(\lambda I-\left[\frac{\kappa_{i} u k_{i}}{\kappa_{i} k_{i}}\right]\right)^{-1}\{1,0,0, \cdots 0\} .
$$

The inverse of a matrix is defined as the quotient of the adjoint of the matrix by its determinant, and the adjoint is the transpose of a matrix whose elements are the cofactors of the corresponding elements in the matrix to be inverted. The determinant of the matrix is already available as $p_{m}(\lambda)$, so it is required to examine the adjoint. Since the adjoint is used to premultiply a column whose elements all vanish except the first, only the first row of the adjoint is needed. The elements of this row will be the cofactors of the elements of the first column in the determinant array of the reduced equation, (13). These cofactors can be written directly by inspection, using polynomials of the form already defined in Eq. (14) but in reverse order, beginning with the lower right corner of the array instead of the upper left. These reversed polynomials will be designated as

$$
\begin{aligned}
\bar{p}_{1}(\lambda) & =\lambda-\alpha_{m}, \\
\bar{p}_{2}(\lambda) & =\left(\lambda-\alpha_{m-1}\right) \bar{p}_{1}(\lambda)-\beta_{m-1}, \\
\bar{p}_{3}(\lambda) & =\left(\lambda-\alpha_{m-2}\right) \bar{p}_{2}(\lambda)-\beta_{m-2} \bar{p}_{1}(\lambda), \\
\bar{p}_{i+1}(\lambda) & =\left(\lambda-\alpha_{m-i}\right) \bar{p}_{i}(\lambda)-\beta_{m-i} \bar{p}_{i-1}(\lambda) .
\end{aligned}
$$

Using these, the first cofactor is $\bar{p}_{m-1}(\lambda)$, the second is $\beta_{1} \bar{p}_{m-2}(\lambda)$, the third is $\beta_{1} \beta_{2} \bar{p}_{m-3}(\lambda)$, and the coefficients, $c_{i}$, may be written, 


$$
\begin{aligned}
& c_{1}=\frac{\bar{p}_{m-1}(\lambda)}{p_{m}(\lambda)}, \\
& c_{2}=\beta_{1} \frac{\bar{p}_{m-2}(\lambda)}{p_{m}(\lambda)}, \\
& c_{3}=\beta_{1} \beta_{2} \frac{\bar{p}_{m-3}(\lambda)}{p_{m}(\lambda)}, \\
& c_{i}=\beta_{1} \beta_{2} \cdots \beta_{i-1} \frac{\bar{p}_{m-i}(\lambda)}{p_{m}(\lambda)} .
\end{aligned}
$$

Applying these to the definition of $k$, the solution is complete.

$$
k=\sum_{i=1}^{m} c_{i} k_{i}=\frac{1}{p_{m}(\lambda)}\left(\bar{p}_{m-1}(\lambda) k_{1}+\beta_{1} \bar{p}_{m-2}(\lambda) k_{2}+\cdots+\beta_{1} \beta_{2} \cdots \beta_{m-1} k_{m}\right) .
$$

5. An outline of the Lanczos algorithm. While the preceding derivation may appear to be complicated, practical application of minimized iterations to numerical calculations requires very simple repeated steps, which are outlined below and which_may easily be translated into operations on automatic computing machinery.

First stage: Given $\kappa_{1}$ and $k_{1}$.

Form $\kappa_{1} u, u k_{1}, \kappa_{1} k_{1}$.

Form $\kappa_{1} u k_{1}$.

$$
\begin{aligned}
& \text { Compute } \alpha_{1}=\frac{\kappa_{1} u k_{1}}{\kappa_{1} k_{1}} . \\
& p_{1}(\lambda)=\lambda-\alpha_{1}=0
\end{aligned}
$$

now yields a first approximation to the first root.

Second stage: Form $\kappa_{2}=\kappa_{1} u-\alpha_{1} \kappa_{1}, k_{2}=u k_{1}-\alpha_{1} k_{1}$.

Form $\kappa_{2} u, u k_{2}, \kappa_{2} k_{2}, \kappa_{2} u k_{2}, \kappa_{1} u k_{2}$.

$$
\begin{aligned}
& \text { Compute } \alpha_{2}=\frac{\kappa_{2} u k_{2}}{\kappa_{2} k_{2}}, \beta_{1}=\frac{\kappa_{1} u k_{2}}{\kappa_{1} k_{1}} \\
& p_{2}(\lambda)=\left(\lambda-\alpha_{2}\right) p_{1}(\lambda)-\beta_{1}=0
\end{aligned}
$$

gives a second approximation to the first root and a first approximation to the second.

Third stage: Form $\kappa_{3}=\kappa_{2} u-\alpha_{2} \kappa_{2}-\beta_{1} \kappa_{1}, k_{3}=u k_{2}-\alpha_{2} k_{2}-\beta_{1} k_{1}$.

Form $\kappa_{3} u, u k_{3}, \kappa_{3} k_{3}, \kappa_{3} u k_{3}, \kappa_{2} u k_{3}$. 


$$
\begin{aligned}
& \text { Compute } \alpha_{3}=\frac{\kappa_{3} u k_{3}}{\kappa_{3} k_{3}}, \beta_{2}=\frac{\kappa_{2} u k_{3}}{\kappa_{2} k_{2}} . \\
& p_{3}(\lambda)=\left(\lambda-\alpha_{3}\right) p_{2}(\lambda)-\beta_{2} p_{1}(\lambda)=0
\end{aligned}
$$

gives the third approximation to the first root, the second approximation to the second root, and a first approximation to the third root. The calculation is continued as far as necessary in continued stages. While the process of minimized iterations as described here can be expected eventually to provide the desired latent roots to any desired degree of accuracy with any initially chosen row and column, the rate of convergence can be made extremely rapid by first multiplying the initial row and column several times by the matrix, $u$, and then beginning the application of the method. The advantage of doing this is that the minimized iterations will then begin with functions whose normal mode components will be "ordered" in the sense that the dominant mode will represent a large part of the initial functions, the second mode will be somewhat smaller but next in importance, the third mode will follow, and so on. To a rough degree of approximation, the orthogonal functions thus generated will then in turn approximate the normal modes in the same order, and the roots of the successive polynomials will also appear in this order. Lanczos [2] presents an impressive numerical example which demonstrates extremely powerful convergence.

It is convenient, particularly in the use of punched card machines, to form certain of the required products simultaneously and to supplement the required operations by check calculations which verify the orthogonality of the generated rows and columns. As an example, consider the third stage. The product, $\kappa_{3} u$, is indicated first and the matrix is bordered by several columns as follows:

$$
\kappa_{3}\left[u, k_{1}, k_{2}, k_{3}\right]=\left[\kappa_{3} u, 0,0, \kappa_{3} k_{3}\right] .
$$

The two zeroes serve as a numerical check. Likewise, the product, $u k_{3}$, is also formed in conjunction with others by bordering $u$ with several rows, as follows:

$$
\left[u, \kappa_{1} u, \kappa_{2} u, \kappa_{3} u\right] k_{3}=\left\{u k_{3}, 0, \kappa_{2} u k_{3}, \kappa_{3} u k_{3}\right\} .
$$

The zero here serves also as a check, and all the scalar products required for the computation of $\alpha_{3}$ and $\beta_{2}$ are available from these operations. In numerical calculations, when the zeroes may occur as small finite numbers due to rounding errors, a refinement is possible by using these numbers to determine the constants, $\gamma_{i}, \delta_{i}, \epsilon_{i}$, etc. of Eq. (7) and (8), which may be employed as indicated by these equations for the generation of further orthogonal functions.

6. Minimized iterations in the Galerkin method. It will now be shown that a minimized iteration technique, similar in form but slightly different in detail, is also applicable to the solution of the eigenvalue problem by the Galerkin method, and that this method may offer certain computational advantages. Beginning with the reduced equation (5) in Galerkin form, but with $k_{j}^{\prime} k_{i}=0$ for $i \neq j$,

$$
\left(\lambda I-\left[\frac{k_{j}^{\prime} u k_{i}}{k_{j}^{\prime} k_{i}}\right]\right) c=0
$$

the generation of suitable columns, $k_{i}$, will be detailed. Using the same technique proposed by Lanczos, let 


$$
k_{i+1}=u k_{i}-\alpha_{i} k_{i}-\beta_{i-1} k_{i-1}-\gamma_{i-2} k_{i-2}-\delta_{i-3} k_{i-3} \cdots
$$

and solve for the constants required on the basis that $k_{j}^{\prime} k_{i}=0(i \neq j)$.

$$
\begin{aligned}
& k_{i}^{\prime} k_{i+1}=k_{i}^{\prime} u k_{i}-\alpha_{i} k_{i}^{\prime} k_{i}=0, \\
& k_{i}^{\prime} k_{i+2}=k_{i}^{\prime} u k_{i+1}-\beta_{i} k_{i}^{\prime} k_{i}=0, \\
& k_{i}^{\prime} k_{i+3}=k_{i}^{\prime} u k_{i+2}-\gamma_{i} k_{i}^{\prime} k_{i}=0,
\end{aligned}
$$

whence

$$
\alpha_{i}=\frac{k_{i}^{\prime} u k_{i}}{k_{i}^{\prime} k_{i}}, \quad \beta_{i}=\frac{k_{i}^{\prime} u k_{i+1}}{k_{i}^{\prime} k_{i}}, \quad \gamma_{i}=\frac{k_{i}^{\prime} u k_{i+2}}{k_{i}^{\prime} k_{i}}, \quad \text { etc. }
$$

However, in the Galerkin case none of these constants vanish. Nevertheless,

and

$$
k_{i+1}^{\prime} u k_{i}=k_{i+1}^{\prime} k_{i+1}
$$

$$
k_{i+j}^{\prime} u k_{i}=0 \quad \text { if } \quad j>1 .
$$

Thus, the reduced matrix becomes

$$
\left[\frac{k_{j}^{\prime} u k_{i}}{k_{i}^{\prime} k_{i}}\right] \equiv\left\|\begin{array}{llllll}
\alpha_{1} & \beta_{1} & \gamma_{1} & \delta_{1} & \cdots & \\
1 & \alpha_{2} & \beta_{2} & \gamma_{2} & \cdots & \\
0 & 1 & \alpha_{3} & \beta_{3} & \cdots & \\
0 & 0 & 1 & \alpha_{4} & \cdots & \\
\cdots & \ldots & \ldots & \ldots & \cdots & \cdots \\
0 & 0 & 0 & 0 & \cdots & \alpha_{m}
\end{array}\right\|,
$$

which is of the same form as Eq. (11), (compare with Eq. (12)), and the polynomial expansions of the determinant by steps are, beginning with the definition,

$$
\begin{aligned}
\left|\lambda I-\left[\frac{k_{j}^{\prime} u k_{i}}{k_{j}^{\prime} k_{i}}\right]\right|=\left|\begin{array}{cccc}
\lambda-\alpha_{1} & -\beta_{1} & -\gamma_{1} & \cdots \\
-1 & \lambda-\alpha_{2} & -\beta_{2} & \cdots \\
0 & -1 & \lambda-\alpha_{3} & \ldots \\
\ldots \ldots \ldots \ldots \ldots \ldots \ldots \ldots \ldots
\end{array}\right|=p_{m}(\lambda) \\
p_{1}(\lambda)=\lambda-\alpha_{1}, \\
p_{2}(\lambda)=\left(\lambda-\alpha_{2}\right) p_{1}(\lambda)-\beta_{1}, \\
p_{3}(\lambda)=\left(\lambda-\alpha_{3}\right) p_{2}(\lambda)-\beta_{2} p_{1}(\lambda)-\gamma_{1}, \\
p_{4}(\lambda)=\left(\lambda-\alpha_{4}\right) p_{3}(\lambda)-\beta_{3} p_{2}(\lambda)-\gamma_{2} p_{1}(\lambda)-\delta_{1} \\
\text { etc. }
\end{aligned}
$$


The roots of these polynomials, as in the Lanczos method, yield successive approximations to the latent roots of the original matrix.

The solution for the coefficients, $c_{i}$, required in order to define the modal columns, must here be accomplished in reverse order, due to the triangular form of the reduced matrix. These coefficients will be expressed in terms of a set of reversed polynomials, defined as follows:

Let

$$
\begin{aligned}
c_{m} & =1, \\
c_{m-1} & =\lambda-\alpha_{m}=\bar{p}_{1}(\lambda), \\
c_{m-2} & =\left(\lambda-\alpha_{m-1}\right) c_{m-1}-\beta_{m-1}=\left(\lambda-\alpha_{m-1}\right) \bar{p}_{1}(\lambda)-\beta_{m-1}=\bar{p}_{2}(\lambda), \\
c_{m-3} & =\left(\lambda-\alpha_{m-2}\right) c_{m-2}-\beta_{m-2} c_{m-1}-\gamma_{m-2}, \\
& =\left(\lambda-\alpha_{m-2}\right) \bar{p}_{2}(\lambda)-\beta_{m-2} \bar{p}_{1}(\lambda)-\gamma_{m-2}=\bar{p}_{3}(\lambda),
\end{aligned}
$$

etc.

Hence, the modal column is written for $\lambda$ corresponding to a root of $p_{m}(\lambda)=0$, in terms of the reversed polynomials defined above.

$$
k=\sum_{i=1}^{m} c_{i} k_{i}=\bar{p}_{m-1}(\lambda) k_{1}+\bar{p}_{m-2}(\lambda) k_{2}+\cdots+\bar{p}_{1}(\lambda) k_{m-1}+k_{m} .
$$

It is interesting to compare Eqs. (21)-(26) with their corresponding relationships in the Lanczos method, Eqs. (6)-(15).

7. The Galerkin treatment of the non-homogeneous equation. In a manner similar to that applied by Lanczos and described in Sec. 4 for the solution of the non-homogeneous equation by minimized iterations, the method of minimized iterations may be extended to the solution of the reduced equation obtained by the Galerkin method. The necessary scalars are determined as described in Sec. 6 , thus permitting the determination of the two sequences of polynomials, $p_{i}(\lambda)$ and $\bar{p}_{i}(\lambda)$. The next step is to solve the reduced equation for the coefficients, $c_{i}$. To accomplish this by determining the cofactors required from the adjoint of the matrix to be inverted is not convenient, in view of the triangular matrix form, but the solution can be easily obtained by first solving for the coefficients in terms of the last, $c_{m}$, working upward in the array until the use of $m-1$ equations provide $c_{1}$ through $c_{m-1}$ in terms of $c_{m}$, and then using the first equation to evaluate $c_{m}$. This process follows:

$$
\begin{aligned}
& c_{m-1}=\left(\lambda-\alpha_{m}\right) c_{m}=\bar{p}_{1}(\lambda) c_{m}, \\
& c_{m-2}=\left(\lambda-\alpha_{m-1}\right) c_{m-1}-\beta_{m-1} c_{m}=\bar{p}_{2}(\lambda) c_{m}, \\
& c_{m-3}=\left(\lambda-\alpha_{m-2}\right) c_{m-2}-\beta_{m-2} c_{m-1}-\gamma_{m-2} c_{m}=\bar{p}_{3}(\lambda) c_{m}, \\
& \quad \text { etc. } \\
& {\left[\left(\lambda-\alpha_{1}\right) \bar{p}_{m-1}(\lambda)-\beta_{1} \bar{p}_{m-2}(\lambda)-\gamma_{1} \bar{p}_{m-3}(\lambda) \cdots\right] c_{m}=1 .}
\end{aligned}
$$


The coefficients having thus been determined, the solution follows directly.

$$
k=\sum_{i=1}^{m} c_{i} k_{i}=\frac{\bar{p}_{m-1}(\lambda) k_{1}+\bar{p}_{m-2}(\lambda) k_{2}+\cdots+\bar{p}_{1}(\lambda) k_{m-1}+k_{m}}{\left(\lambda-\alpha_{1}\right) \bar{p}_{m-1}(\lambda)-\beta_{1} \bar{p}_{m-2}(\lambda)-\gamma_{1} \bar{p}_{m-3}(\lambda) \cdots} .
$$

In view of the means by which the $c_{i}$ were determined, Eq. (27) does not closely resemble Eq. (20).

8. An outline of the minimized iteration algorithm in the Galerkin method. Again it is desirable to present an outline of the actual steps in numerical calculation by minimized iterations, from which a logical sequence of operations may be established for purposes of automatic calculation.

First stage: Given $k_{1}$.

Form $u k_{1}, k_{1}^{\prime} k_{1}$.

Form $k_{1}^{\prime} u k_{1}$.

Compute $\alpha_{1}=\frac{k_{1}^{\prime} u k_{1}}{k_{1}^{\prime} k_{1}}$.

$p_{1}(\lambda)=\lambda-\alpha_{1}$

Second stage: Form $k_{2}=u k_{1}-\alpha_{1} k_{1}$.

Form $u k_{2}, k_{2}^{\prime} k_{2}, k_{2}^{\prime} u k_{2}, k_{1}^{\prime} u k_{2} \cdot$.

Compute $\alpha_{2}=\frac{k_{2}^{\prime} u k_{2}}{k_{2}^{\prime} k_{2}}, \beta_{1}=\frac{k_{1}^{\prime} u k_{2}}{k_{1}^{\prime} k_{1}}$.

$p_{2}(\lambda)=\left(\lambda-\alpha_{2}\right) p_{1}(\lambda)-\beta_{1}$.

Third stage: Form $k_{3}=u k_{2}-\alpha_{2} k_{2}-\beta_{1} k_{1}$.

Form $u k_{3}, k_{3}^{\prime} k_{3}, k_{3}^{\prime} u k_{3}, k_{2}^{\prime} u k_{3}, k_{1}^{\prime} u k_{3}$.

Compute $\alpha_{3}=\frac{k_{3}^{\prime} u k_{3}}{k_{3}^{\prime} k_{3}}, \beta_{2}=\frac{k_{2}^{\prime} u k_{3}}{k_{2}^{\prime} k_{2}}, \gamma_{1}=\frac{k_{1}^{\prime} u k_{3}}{k_{1}^{\prime} k_{1}}$.

$p_{3}(\lambda)=\left(\lambda-\alpha_{3}\right) p_{2}(\lambda)-\beta_{2} p_{1}(\lambda)-\gamma_{1}$.

As in the Lanczos procedure described in Sec. 5, it is advantageous to precede this process with several premultiplications of an arbitrary column in order to control the relative magnitudes of the various modal components of the column, $k_{1}$, so that the roots will be obtained from the successive polynomials in order of relative magnitudes. As indicated for the Lanczos algorithm, it is convenient in punched card procedures to form certain of the required products simultaneously and to include check calculations. 
To this end the matrix, $u$, is bordered by a sequence of rows, as exemplified below for the third stage.

$$
\left[u, k_{1}^{\prime}, k_{2}^{\prime}, k_{3}^{\prime}\right] k_{3}=\left\{u k_{3}, 0,0, k_{3}^{\prime} k_{3}\right\} .
$$

The two zeroes serve as a check on the orthogonality of the generated columns. In addition, the following operations are necessary:

$$
\left[k_{3}^{\prime}, k_{2}^{\prime}, k_{1}^{\prime}\right] u k_{3}=\left\{k_{3}^{\prime} u k_{3}, k_{2}^{\prime} u k_{3}, k_{1}^{\prime} u k_{3}\right\} .
$$

whence the constants, $\alpha_{3}, \beta_{2}$, and $\gamma_{1}$, may be calculated.

9. General comments on minimized iterations. It should be noted that the foregoing presentation and extension of the method originally proposed by Lanczos has leaned toward the pictorial approach to a means of mathematical analysis rather than concerning itself with important considerations of mathematical rigor. The special cases of equal roots and of unfortunate choice of arbitrary row or column, where a deficiency in modal components might lead to peculiarities in the results, have been deliberately ignored, first, because these cases are adequately treated in Lanczos' paper [2], and second, because they would detract from the object of presenting a practical procedure and of emphasizing the computational aspects which are of interest to the applied mathematician and to the engineer. Furthermore, in the interest of providing a simple derivation which would admit of obvious extension to the Galerkin type equation, the original intent of the name, minimized iterations, was passed over lightly, deriving the basic scalars through strictly algebraic operations without consideration of their more fundamental purpose of providing the maximum utility with the minimum number of row and column functions. The advantages of orthogonal functions in computation provided an equally direct reason for the choice of these scalars.

The method of minimized iterations, in either the Lanczos or the Galerkin variations, is recommended as an alternative to conventional procedures in the numerical solution of matrix problems on the basis of potentially large time-saving in computation. Its advantages are most evident when the determination of a number of eigenvalues, or latent roots, is of prime interest, and the determination of modal columns or the solution of non-homogeneous equations can also be greatly expedited, subject to an efficient organization of problems for whatever type of computing machinery may be available.

The Galerkin variation appears to be of greatest advantage in the eigenvalue problem, requiring substantially only half the number of matrix-column products involved in the Lanczos procedure. However, the computation of modal columns by this method is somewhat more cumbersome, particularly if successive approximations to a modal column are to be studied, in view of the requirement for reversed polynomials. In the non-homogeneous equation, on the other hand, this disadvantage applies to both versions, the only significant difference being that the Galerkin polynomials are longer.

\section{RefERENCES}

1. A. C. Aitken, Studies in practical mathematics. I. The evaluation, with applications, of a certain triple product matrix, Proc. Roy. Soc. Edinburgh, 57, 172-181 (1937).

2. C. Lanczos, An iteration method for the solution of the eigenvalue problem of linear differential and integral operators, Journal of Research, Nat. Bu. Stand., 45, 255-282 (1950).

3. W. J. Duncan, Galerkin's method in mechanics and differential equations, R. \& M. 1798 (1938). 